\begin{tabular}{c|c|c|} 
SELECCIONES MATEMÁTICAS \\
Universidad Nacional de Trujillo \\
ISSN: $2411-1783$ (Online) \\
2021; Vol. 8(1): 147-160.
\end{tabular}

\title{
A Leslie-Gower-type predation model considering generalist predators and the Allee effect on prey
}

\section{Un modelo de depredación del tipo Leslie-Gower considerando depredadores generalistas y efecto Allee en las presas}

Nicole Martínez-Jeraldo (), Elizabeth Rozas-Torres (i) and Eduardo González-Olivares (i)

Received, Mar. 29, 2021

Accepted, Jun. 30, 2021

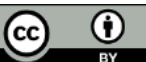

How to cite this article:

Martínez-Jeraldo N, et al. A Leslie-Gower-type predation model considering generalist predators and the Allee effect on prey. Selecciones Matemáticas. 2021;8(1):147-160. http://dx.doi.org/10.17268/sel.mat.2021.01. 14

\begin{abstract}
The main feature of the Leslie-Gower-type predation model is that the predator's growth function is one of logistic-type. Thus, it is a model assuming implicitly the competition among predators.

In this work the dynamics of a modified Leslie-Gower type predator-prey model is analyzed, considering two important aspects: (i) the predators capture an alternative food when the quantity of prey is scarce and (ii) the prey population is affected by an Allee effect.

Considering a topological equivalent system, the main properties of the system are established. Necessary and sufficient conditions for the existence and local stability of equilibria are determined, also showing the existence of a homoclinic orbit and of at least a limit cycle.

When the predators are generalists the dynamics of the model differ enough respecting the model considering predators specialist since appearing more equilibrium points and the mentioned homoclinic orbit.
\end{abstract}

Keywords . Predator-prey model, bifurcation, limit cycles, separatrix curve, stability, functional response.

\section{Resumen}

La característica principal de los modelos del tipo Leslie-Gower, es que la ecuación de crecimiento de los depredadores es descrita por la función de logística. Por lo tanto, es un modelo que supone implícitamente la competencia entre los depredadores.

En este trabajo se analiza la dinámica de un modelo derivado del modelo de Leslie-Gower, considerando dos aspectos importantes: (i) los depredadores capturan un alimento alternativo cuando la cantidad de presas es escasa y (ii) la población de presas se ve afectada por un efecto Allee.

Considerando un sistema equivalente topológico, se establecen las principales propiedades del modelo. Se determinan las condiciones necesarias y suficientes para la existencia y la estabilidad local de los equilibrios. Además, se prueba la existencia de una órbita homoclínica y de al menos un ciclo límite.

Cuando los depredadores son generalistas, la dinámica del modelo difiere bastante con respecto al modelo donde los depredadores son especialistas. Dinámicamente aparecen más puntos de equilibrio y una órbita homoclínica. nal.

Palabras clave. Modelo depredador-presa, bifurcación, ciclo límite, curva separatriz, estabilidad, respuesta funcio-

\footnotetext{
* Doctorado Modelamiento Matemático Aplicado, Universidad Católica del Maule, Talca, Chile. (ni cole.m.j05 @ gma il . com).

${ }^{\dagger}$ Centro Docencia de Ciencias Básicas para Ing. Universidad Austral de Chile, Valdivia, Chile. (elizabeth.rozas@uach.cl).

${ }^{\ddagger}$ Pontificia Universidad Católica de Valparaíso, Chile. (e jgonzal@ucv. cl).
} 
1. Introducción. La interacción entre los depredadores y sus presas es uno de los temas dominantes en la Ecología Teórica debido a su existencia universal [9], tanto en nivel microscópico como macroscópico. Particularmente en Dinámica Poblacional, la comprensión de esta importante relación permite un mejor estudio y análisis del comportamiento de las cadenas alimentarias o redes tróficas [35, 43].

A partir del primer modelo para la depredación, propuesto por el el matemático italiano Vito Volterra [45], se han sucedido una gran cantidad de modelos para este comportamiento de ciertas especies. En principio estas nuevas proposiciones trataban de resolver las variadas objeciones formuladas al primer modelo, descrito por un sistema de ecuaciones diferenciales ordinarias (EDO) no lineales autónomo. Este sistema es conocido como modelo depredador-presa de Lotka-Volterra [7, 43].

En 1948 Patrick H. Leslie [31], formuló un nuevo modelo para responder algunas de las críticas formuladas contra el modelo Lotka-Volterra. Su proposición no se ajusta al esquema de dicho modelo [43], sendo la principal diferencia que la ecuación de crecimiento de los depredadores se basa en la bien conocida función logística. Entonces, el modelo supone implícitamente la competencia intraespecífica entre los depredadores [32]. Pero el modelo mantiene la ecuación para las presas del modelo Volterra [43], en el que también se supone la autointerferencia entre las presas; además, la isoclina de las presas es oblicua [43].

Entonces, el modelo depredador-presa de tipo Leslie o Leslie-Gower es descrito por un sistema autónomo bidimensional de ecuaciones diferenciales no-lineales caracterizado por:

i) la respuesta funcional o tasa de consumo de los depredadores es lineal [7, 19, 35, 43], y

ii) la ecuación para los depredadores es una función de crecimiento de tipo logístico [1, 36, 43], donde la capacidad de carga del medio ambiente convencional para los depredadores $K_{y}$ es proporcional a la abundancia de presas $x=x(t)$, esto es, $K_{y}=K(x)=n x[35,43]$.

Si $y=y(t)$ representa el tamaño de la población de depredadores en el modelo logístico de depredadores, el cociente $\left(\frac{y}{n x}\right)$ se llama el término de Leslie-Gower, que mide la pérdida en la población de depredadores debido a la rareza de su comida favorita.

En el caso de escasez severa, algunos depredadores pueden cambiar a otra población de presas, aunque su crecimiento estaría limitado por el hecho de que su alimento favorito no está disponible en abundancia. La capacidad de carga ambiental para los depredadores puede describirse por $K(x)=n x+c$, con $c>0$.

En ese caso, se dice que el modelo está representado por un esquema de Leslie-Gower, también conocido como modelo de Leslie-Gower modificado [4, 29]. Si $x=0$, entonces $K(0)=c$, concluyendo que los depredadores son generalistas ya que buscan un alimento alternativo, evitando su extinción.

En este trabajo se analiza un modelo depredador-presa del tipo Leslie-Gower modificado, considerando que:

(i) los depredadores capturan un alimento alternativo cuando la cantidad de presas es escasa y

(ii) la población de presas se ve afectada por un efecto Allee.

Se dice que una población está bajo el efecto Allee si la tasa de crecimiento per cápita es inicialmente una función creciente, luego disminuye a cero a una densidad más alta [16, 17, 41, 42]. Para algunas poblaciones, la tasa de crecimiento no siempre es positiva para un tamaño de población pequeña y puede no estar disminuyendo como lo sugiere el modelo logístico, lo que genera una relación positiva entre la densidad de población y la aptitud (fitness) del individuo [16, 17, 41, 42].

En este trabajo, empleamos la forma matemática más común para expresar la tasa de crecimiento de una población afectada por el efecto Allee, que se describe mediante la ecuación diferencial polinómica cúbica $[5,44]$

$$
\frac{d x}{d t}=r\left(1-\frac{x}{K}\right)(x-m) x .
$$

El efecto Allee es un fenómeno ecológico que ocurre a bajos tamaños de población, donde la tasa de crecimiento per cápita es una función creciente de la abundancia de la población, cuando el tamaño de la población s bajo. En tamaños de población grandes, esta tasa es negativa, como ocurre en la ecuación logística para todos los tamaños de población. Por esta razón, la ecuación (1.1) representa un efecto Allee, si y solo si, $-K<m<<K, \operatorname{con} r, K>0$.

Cuando $m>0$, la tasa de crecimiento de la población disminuye si el tamaño de la población está por debajo del nivel umbral $m$ y la población se extingue. En este caso, la ecuación (1.1) describe un efecto Allee fuerte $[44,46]$.

Si $m \leq 0$, se dice que la población se ve afectada por un efecto Allee débil [26]. En Ciencias Pesqueras, los mismos fenómenos se denominan dependensación crítica y pura, respectivamente [14].

Este efecto puede deberse a una amplia gama de fenómenos biológicos, tales como: depresión endogámica, escasez de alimentos, búsqueda de pareja, disfunción social, defensa anti-depredadores y otros (ver Tabla 1 en [8] o la Tabla 2.1 en [17]). 
El efecto Allee puede ser modelados por variadas formas algebraicas [1, 2, 34], pero es posible demostrar que muchas de ellas son topológicamente equivalentes [23]. No obstante, las diferentes formas pueden producir cambios en las dinámicas de los sistemas, particularmente en la cantidad de ciclos límites que rodean un punto de equilibrio positivo en los modelos depredador-presa [1, 2, 26, 40].

Además, dos o más efectos Allee producidos por diferentes causas pueden generar mecanismos que actúan simultáneamente en una sóla población (consulte la Tabla 2 en [8] o la Tabla 2.2 en [17]). La influencia combinada de algunos de estos fenómenos se denomina efecto múltiple Allee [8].

En este trabajo extendemos los resultados obtenidos en [5], mostrando la existencia de una curva homoclínica. Cuando se rompe esa curva homoclínica, se obtiene un ciclo límite no infinitesimal cuya estabilidad se determina calculando el valor absoluto del cociente entre los valores propios negativos y positivos de los puntos sillas $[3,7]$.

Este artículo está organizado de la siguiente manera: en la Sección 2, presentamos el modelo y, por medio de un difeomorfismo, se obtiene un modelo topológicamente equivalente al modelo depredador-presa del tipo Leslie-Gower; en la Sección 3, se presentan las principales propiedades del sistema. Algunas consecuencias ecológicas y una discusión de los resultados matemáticos se dan en la Sección 4.

2. El modelo. El modelo modificado Leslie-Gower considerando el efecto Allee viene dado por el siguiente sistema de ecuaciones diferenciales bidimensionales del tipo Kolmogorov [19, 22]:

$$
X_{\nu}(x, y):\left\{\begin{array}{rl}
\frac{d x}{d t} & =\left(r\left(1-\frac{x}{K}\right)(x-m)-q y\right) x, \\
\frac{d y}{d t}=s\left(1-\frac{y}{n x+c}\right) y
\end{array} .\right.
$$

donde $x=x(t)$ e $y=y(t)$ indican los tamaños de población de presas y depredadores, respectivamente, para $t \geq 0$, medida como número de individuos, biomasa, o bien, densidad por unidad de área o volumen, con $\left.\nu=(r, K, q, s, n, c, m) \in \mathbb{R}_{+}^{5} \times\left(\mathbb{R}_{+} \cup\{0\}\right) \times\right]-K, K[$, que tienen los siguientes significados:

$r$ es la tasa de crecimiento intrínseca de presas o potencial biótico,

$K$ es la capacidad de carga ambiental de la presa,

$q$ es la tasa de captura de los depredadores,

$s$ es la tasa de crecimiento intrínseca de los depredadores,

$n$ es una medida de la calidad de los alimentos que indica cómo los depredadores convierten a las presas consumidas en nuevos nacimientos de depredadores,

$c$ es la cantidad de comida alternativa disponible para los depredadores.

Este último parámetro indica que los depredadores son generalistas y que si su presa no eatá disponible, tiene una fuente de alimento alternativo. Claramente, si $c=0$, la capacidad de carga del depredador es $K(x)=n x$ y el modelo Leslie-Gower no está definido en $x=0$. Advertimos que se ha dado otro significado para este parámetro, indicando que mide el grado en que el entorno proporciona protección al depredador.

El sistema (2.1) o el campo vectorial $X_{\nu}$ se define en

$$
\Omega=\left\{(x, y) \in \mathbb{R}^{2} \mid x \geq 0, y \geq 0\right\}=\mathbb{R}_{0}^{+} \times \mathbb{R}_{0}^{+} .
$$

Los puntos de equilibrio del sistema (2.1) o del campo vectorial $X_{\mu}$ son $(0,0),(K, 0),(0, c),(m, 0)$ cuando $m>0$, y $\left(x_{e}, y_{e}\right)$ satisfaciendo la ecuación de las isoclinas $y=n x+c$ e $y=\frac{r}{q}\left(1-\frac{x}{K}\right)(x-m)$.

Claramente, $\left(x_{e}, y_{e}\right)$ puede ser un punto de equilibrio positivo (equilibrio en el interior del primer cuadrante) o no puede existir allí, dependiendo del signo de los factores $1-\frac{x}{K}$ y $x-m$.

Existen versiones no autónomas del sistema (2.1) incorporando retardos, impulsos, como también usando derivadas parciales. A pesar de estos estudios recientes, la dinámica del sistema (2.1) no se ha analizado completamente y algunos resultados existentes no son verdaderos o no se comprenden bien.

2.1. Equivalencia topológica. Para simplificar los cálculos, seguimos la metodología utilizada en [4, 24, 28, 36, 40], que se describe en el siguiente lema.

Lema 2.1. El sistema (2.1) es topológicamente equivalente al sistema de Kolmogorov [19, 22]

$$
Y_{\eta}(u, v):\left\{\begin{array}{rl}
\frac{d x}{d t} & =((1-u)(u-M)-Q v)(u+C) u \\
\frac{d y}{d t} & =S(u+C-v) v
\end{array} .\right.
$$

donde $\left.\eta=(Q, C, S, M) \in \mathbb{R}_{+}^{3} \times\right]-1,1\left[\operatorname{con} Q=\frac{q c}{r}, C=\frac{c}{n K}, S=\frac{s}{r K} y M=\frac{m}{K}\right.$. 
Demostración: Usando el cambio de variables dado por $x=K u$ e $y=n K v$, reemplazando en el sistema (2.1) y luego de una simplificación, obtenemos el nuevo campo vectorial

\section{Factorizando se obtiene}

$$
U_{\nu}(u, v):\left\{\begin{aligned}
K \frac{d u}{d t} & =(r(1-u)(K u-m)-q n K v) K u \\
n K \frac{d v}{d t} & =s\left(1-\frac{n K v}{n K u+c}\right) n K v
\end{aligned}\right.
$$

$$
U_{\nu}(u, v):\left\{\begin{array}{l}
\frac{d u}{d t}=r K\left((1-u)\left(u-\frac{m}{K}\right)-\frac{q n}{r} v\right) u, \\
\frac{d v}{d t}=s\left(1-\frac{v}{u+\frac{c}{n K}}\right) v
\end{array} .\right.
$$

Mediante el reescalamiento de tiempo dado por $\tau=\frac{r K}{u+\frac{c}{n K}} t$, y usando la regla de la cadena, obtenemos:

$$
\frac{d u}{d t}=\frac{d u}{d \tau} \tau d t=\frac{d u}{d \tau} \frac{r K}{u+\frac{c}{n K}} \text { y } \frac{d v}{d t}=\frac{d v}{d \tau} \frac{r K}{u+\frac{c}{n K}} .
$$

Entonces, tenemos el sistema

llegando

$$
V_{\nu}(u, v):\left\{\begin{array}{l}
\frac{r K}{u+\frac{c}{n K}} \frac{d u}{d \tau}=r K\left((1-u)\left(u-\frac{m}{K}\right)-\frac{q n}{r} v\right) u, \\
\frac{r K}{u+\frac{c}{n K}} \frac{d v}{d \tau}=s\left(1-\frac{v}{u+\frac{c}{n K}}\right) v .
\end{array}\right.
$$

$$
V_{\nu}(u, v):\left\{\begin{aligned}
\frac{d u}{d \tau} & =\left((1-u)\left(u-\frac{m}{K}\right)-\frac{q n}{r} v\right)\left(u+\frac{c}{n K}\right) u \\
\frac{d v}{d \tau} & =\frac{s}{r K}\left(u+\frac{c}{n K}-v\right) v
\end{aligned}\right.
$$

Definiendo $Q=\frac{q c}{r}, C=\frac{b}{c K}, S=\frac{s}{r K}$ y $M=\frac{m}{K}$, se obtiene el sistema (2.2).

Observación 2.1. Hemos construido el difeomorfismo $[13,18] \varphi: \tilde{\Omega} \times \mathbb{R} \longrightarrow \Omega \times \mathbb{R}$, tal que

$$
\varphi(u, v, \tau)=\left(K u, n K v, \frac{u+\frac{c}{n K}}{r K} \tau\right)=(x, y, t),
$$

donde $\tilde{\Omega}=\left\{(u, v) \in \mathbb{R}^{2} \mid u \geq 0, v \geq 0\right\}$

con

$$
\operatorname{det} D \varphi(u, v, \tau)=\operatorname{det}\left(\begin{array}{ccc}
K & 0 & 0 \\
0 & n K & 0 \\
\frac{1}{r K} & 0 & \frac{1}{r K}\left(u+\frac{c}{n K}\right)
\end{array}\right)=\frac{n K}{r}\left(u+\frac{c}{n K}\right)>0 .
$$

Entonces, $\varphi$ es un diffeomorfismo que preserva la orientación del tiempo. El campo vectorial $X_{\nu}$ en el nuevo sistema de coordenadas es topológicamente equivalente al campo vectorial $Y_{\eta}=\varphi \circ X_{\nu}$, que tiene la forma $Y_{\eta}=P(u, v) \frac{\partial}{\partial u}+Q(u, v) \frac{\partial}{\partial v}$ [18], siendo $P(u, v)$ y $Q(u, v)$ el lado derecho de las ecuaciones respectivas en el sistema (2.2).

El campo vectorial $Y_{\eta}(u, v)$ es una extensión continua del sistema (2.2) definido en $\tilde{\Omega}$.

Nuestro análisis se realizará considerando diferentes casos para $M>0, M=0$ y $M<0$.

2.2. Puntos de equilibrio. Los puntos de equilibrio del sistema (2.2) o las singularidades del campo vectorial $Y_{\eta}$ son $(1,0),(0,0),(M, 0)$ cuando $M>0,(0, C)$ y los puntos $\left(u_{e}, v_{e}\right)$ están en la intersección de curvas

$$
v=\frac{1}{Q}(1-u)(u-M) \text { y } v=u+C .
$$

Entonces, la abscisa $u_{e}$ de los puntos de equilibrio positivo es la solución de la ecuación de segundo grado:

$$
p(u)=u^{2}-(1+M-Q) u+(C Q+M)=0 .
$$

Considerando la regla de los signos de Descartes y de acuerdo con el signo del coeficiente $a_{1}=1+M-$ $Q$, la ecuación (2.3) tiene dos, una o ninguna raíz positiva.

El coeficiente $a_{0}=C Q+M>0$, si y solo si, $M \geq 0$. Pero $a_{0}$ puede ser negativo si $M<0 \mathrm{y}$ $C Q<-M$.

Caso 1. Suponiendo que $M>0$.

1. Suponiendo que $a_{1}>0$, entonces la ecuación (2.3) tiene: 
(a) dos raíces reales positivas, si y solo si, $\Delta=(1+M-Q)^{2}-4(C Q+M)>0$, que son:

$$
u_{1}=\frac{1}{2}(1+M-Q-\sqrt{\Delta}) \text { y } u_{2}=\frac{1}{2}(1+M-Q+\sqrt{\Delta}),
$$

con $u_{1}<u_{2}$.

(b) una raíz real positiva, si y solo si, $\Delta=0$, que es dado por

$$
u^{*}=\frac{1}{2}(1+M-Q) .
$$

(c) ninguna raíz real positivas, si y solo si, $\Delta<0$.

2. ninguna raíz real positivas, si y solo si, $1+M-Q \leq 0$.

Caso 2. Suponiendo que $M=0$.

Los puntos de equilibrio del sistema (2.2) son $(1,0),(0,0),(0, C)$ (el punto $(M, 0)$ coincide con $(0,0)$ ) y los puntos $\left(u_{e}, v_{e}\right)$, con $u_{e}$ satisfaciendo la ecuación (2.3) que se reduce a

$$
p(u)=u^{2}-(1-Q) u+C Q=0
$$

La ecuación tiene:

1. Asumiendo $b_{1}=1-Q>0$, la ecuación (2.4) tiene

(a) dos raíces reales positivas, si y solo si, $\Delta_{0}=(1-Q)^{2}-4 C Q>0$, i.e. $C<\frac{(1-Q)^{2}}{4 Q}$, que son:

$$
u_{3}=\frac{1}{2}(1-Q-\sqrt{\Delta}) \text { and } u_{4}=\frac{1}{2}(1-Q+\sqrt{\Delta})
$$

con $u_{3}<u_{4}$.

(b) una raíz real positiva, si y solo si, $\Delta_{0}=0$, que es dado por

$$
u^{* *}=\frac{1}{2}(1-Q) \text {. }
$$

(c) ninguna raíz real positiva, si y solo si, $\Delta_{0}<0$.

2. ninguna raíz real positiva, si y solo si, $b_{1}=1-Q \leq 0$.

Case 3. Suponiendo que $M<0$.

En este caso $(M, 0)$ está en el segundo cuadrante y se tiene las siguientes alternativas

1. Cuando $C Q+M>0$, tiene las mismas posibilidades que el caso 1, es decir, hay dos, una o ninguna raíz real positiva.

2. Cuando $C Q+M=0$, la ecuación (2.3) se reduce

$$
p(u)=\left(u^{2}-(1+M-Q)\right) u=0 .
$$

Luego,

(a) $\mathrm{Si} c_{1}=1+M-Q>0$, la única raíz real positiva es $u_{5}=1+M-Q$, (la solución $u=0$ se repite).

(b) Si $c_{1}=1+M-Q \leq 0$, no existe una raíz real positiva.

3. Si $C Q+M<0$,

(a) Si $c_{1}=1+M-Q \neq 0$, cualquiera sea el signo de $1+M-Q$, la ecuación $(2,5)$ tiene una raíz real positiva dada por

$$
u_{6}=\frac{1}{2}((1+M-Q)+\sqrt{\Delta}) .
$$

(b) Si $c_{1}=1+M-Q=0$, la ecuación (2.5) se reduce a

$$
p(u)=u^{2}+(C Q+M)=0
$$

que tiene una raíz real positiva única, dada por $u_{7}=\sqrt{(C Q+M)}$.

Para determinar la naturaleza local de los puntos de equilibrio debemos obtener la matriz jacobiana del sistema (2.2), la cual es: 


$$
D Y_{\eta}(u, v)=\left(\begin{array}{cc}
D Y_{\eta}(u, v)_{11} & -Q u(C+u) \\
S v & S(-2 v+u+C)
\end{array}\right),
$$

con

$$
\begin{aligned}
D Y_{\eta}(u, v)_{11} & =\frac{d}{d u}(((1-u)(u-M)-Q v)(u+C) u) \\
& =((1-u)(u-M)-Q v)(u+C)+((1-u)(u-M)-Q v) u+(u+C) u(M-2 u+1) .
\end{aligned}
$$

3. Resultados Principales . Para el sistema (2.2) tenemos las siguientes propiedades generales:

Lema 3.1. Existencia de una región positivamente invariante [13].

El conjunto $\tilde{\Gamma}=\{(u, v) \in \tilde{\Omega} / 0 \leq u \leq 1, v \geq 0\}$ es una región positivamente invariante.

Demostración: Claramente, el eje $-u$ y el $e j e-v$ son conjuntos invariables porque el sistema es del tipo Kolmogorov. Si $u=1$, tenemos

$$
\frac{d u}{d \tau}=-Q v(1+C)<0
$$

y cualquiera que sea el signo de

$$
\frac{d v}{d \tau}=S(1+C-v) v,
$$

las trayectorias entran y permanecen en la región $\tilde{\Gamma}$.

Lema 3.2. Acotamiento de las soluciones.

Las soluciones son acotadas

Demostración:

Usando compactificación de Poincaré [38], sea

$$
X=\frac{u}{v} \quad Y=\frac{1}{v} .
$$

Asi tenemos que

$$
\frac{d X}{d \tau}=\left(\frac{d u}{d \tau} v-\frac{d v}{d \tau} u\right) \frac{1}{v^{2}} \quad \frac{d Y}{d \tau}\left(-\frac{d v}{d \tau}\right) \frac{1}{v^{2}} .
$$

Luego el sistema (2.2) queda

$$
\widehat{\Gamma}_{\eta}(X, Y):\left\{\begin{aligned}
\frac{d X}{d \tau} & =-\frac{X}{Y^{3}}\left(X^{3}-X^{2} Y-S Y^{2}+C M Y^{3}+C Q Y^{2}+C S Y^{3}-C X Y^{2}\right. \\
& \left.+C X^{2} Y+M X Y^{2}-M X^{2} Y+S X Y^{2}+Q X Y-C M X Y^{2}\right), \\
\frac{d Y}{d \tau} & =-S(X+C Y-1) .
\end{aligned}\right.
$$

Efectuando un reescalamiento del tiempo $T=\frac{1}{Y^{3}} \tau$, con $\frac{d T}{d \tau}=\frac{1}{Y^{3}}, \frac{d X}{d \tau}=\frac{d X}{d T} \frac{d T}{d \tau}, \frac{d Y}{d \tau}=\frac{d Y}{d T} \frac{d T}{d \tau}$ tenemos que el campo vectorial $\widehat{\Gamma}_{\eta}(X, Y)$ es topologicamente equivalente al siguiente sistema

$$
\bar{\Gamma}_{\eta}(X, Y):\left\{\begin{array}{l}
\frac{d X}{d T}=-X\left(X^{3}-X^{2} Y-S Y^{2}+C M Y^{3}+C Q Y^{2}+C S Y^{3}-C X Y^{2},\right. \\
\left.+C X^{2} Y+M X Y^{2}-M X^{2} Y+S X Y^{2}+Q X Y-C M X Y^{2}\right) \\
\frac{d Y}{d T}=-S Y^{3}(X+C Y-1) .
\end{array}\right.
$$

Luego vemos que

$$
D \bar{\Gamma}_{\eta}(0,0)=\left(\begin{array}{ll}
0 & 0 \\
0 & 0
\end{array}\right) .
$$

Para desingularizar el origen, aplicamos el metodo de Blowing up [18], haciendo los siguientes cambios $X=r, Y=r^{2} p, \frac{d X}{d T}=\frac{d r}{d T}, \frac{d p}{d T}=\left(\frac{d Y}{d T}-\frac{d r}{d T} 2 r p\right) \frac{1}{r^{2}}$. Luego el campo vectorial $\bar{\Gamma}_{\eta}(X, Y)$ nos queda

$$
\bar{\Gamma}_{\eta}(p, r):\left\{\begin{array}{c}
\frac{d r}{d T}=-r^{4}\left(Q p-r p-S r p^{2}-C r^{2} p^{2}+M r^{2} p^{2}+S r^{2} p^{2}+C r p-M r p+C Q r p^{2}\right. \\
\left.\quad-C M r^{2} p^{2}+C M r^{3} p^{3}+C S r^{3} p^{3}+1\right), \\
\frac{d p}{d T}=r^{3} p\left(2 Q p-2 r p-S r p^{2}-2 C r^{2} p^{2}+2 M r^{2} p^{2}+S r^{2} p^{2}+2 C r p-2 M r p+2 C Q r p^{2}\right. \\
\left.-2 C M r^{2} p^{2}+2 C M r^{3} p^{3}+C S r^{3} p^{3}+2\right) .
\end{array}\right.
$$


Para simplificar el sistema, hacemos un reescalamiento del tiempo dado por $\lambda=r^{3} T, \frac{d r}{d T}=\frac{d r}{d \lambda} \frac{d \lambda}{d T}$, $\frac{d p}{d T}=\frac{d p}{d \lambda} \frac{d \lambda}{d T}$, obtiendo un sistema topologicamente equivalente al campo vectorial $\bar{\Gamma}_{\eta}(p, r)$

$$
\widehat{F}_{\eta}(p, r):\left\{\begin{aligned}
\frac{d r}{d \lambda}= & -r\left(Q p-r p-S r p^{2}-C r^{2} p^{2}+M r^{2} p^{2}+S r^{2} p^{2}+C r p-M r p+C Q r p^{2}\right. \\
& \left.-C M r^{2} p^{2}+C M r^{3} p^{3}+C S r^{3} p^{3}+1\right) \\
\frac{d p}{d \lambda}= & p\left(2 Q p-2 r p-S r p^{2}-2 C r^{2} p^{2}+2 M r^{2} p^{2}+S r^{2} p^{2}+2 C r p-2 M r p+2 C Q r p^{2}\right. \\
& \left.-2 C M r^{2} p^{2}+2 C M r^{3} p^{3}+C S r^{3} p^{3}+2\right)
\end{aligned}\right.
$$

Luego tenemos que

$$
D \widehat{F}_{\eta}(0,0)=\left(\begin{array}{cc}
-1 & 0 \\
0 & 2
\end{array}\right)
$$

por lo que $\operatorname{det}\left(D \widehat{F}_{\eta}(0,0)\right)=-2<0$, y el punto $(0,0)$ es punto silla del campo vectorial $\widehat{F}_{\eta}(p, r)$, por lo cual el punto $(0, \infty)$ es punto silla del campo vectorial compactificado $\widehat{\Gamma}_{\eta}$.

Por lo tanto las soluciones son acotadas.

3.1. Modelo con efecto Allee fuerte. En lo siguiente asumimos $1>M>0$ y $C>0$.

3.1.1. Naturaleza de los puntos de equilibrio sobre los ejes. Para las dingularidades sobre los ejes tenemos las siguientes propiedades

Lema 3.3. Las singularidades $(0,0)$ y $(1,0)$ son puntos silla hiperbólicos [6], para todo $\eta=(Q, C, S, M) \in$ $\left.\mathbb{R}_{+}^{3} \times\right] 0,1[$.

Demostración: Evaluando la matriz Jacobiana en cada punto tenemos

1. $D Y_{\eta}(0,0)=\left(\begin{array}{cc}-C M & 0 \\ 0 & S C\end{array}\right)$,

$\mathrm{y} \operatorname{det} D Y_{\eta}(0,0)=-S C^{2} M<0$.

Entonces, de acuerdo al Teorema de la traza y el determinante, el equilibrio $(0,0)$ es un punto silla.

2. $D Y_{\eta}(1,0)=\left(\begin{array}{cc}-(1+C)(1-M) & -Q(1+C) \\ 0 & S(1+C)\end{array}\right)$,

y det $D Y_{\eta}(1,0)=-S(1+C)^{2}(1-M)<0$.

Por lo tanto, el equilibrio $(1,0)$ es un punto silla, aplicando el Teorema de la traza y el determinante.

Lema 3.4. El punto de equilibrio $(M, 0)$ es un repulsor hiperbólico [6] para todo los valores de los parámetros.

Demostración: Es inmediato, ya que la matriz jacobiana evaluada en $(M, 0)$ es

Por lo tanto,

$$
D Y_{\eta}(M, 0)=\left(\begin{array}{cc}
M(1-M)(C+M) & -Q M(C+M) \\
0 & S(C+M)
\end{array}\right)
$$

$\operatorname{det} D Y_{\eta}(M, 0)=M(1-M)(C+M)^{2}>0 \mathrm{y}$

$\operatorname{tr} D Y_{\eta}(M, 0)=(M(1-M)+S)(C+M)>0$. Aplicando el Teorema de la traza y el determinante se tiene la tesis.

Lema 3.5. El equilibrio $(0, C)$ es un punto atractor hiperbólico [6], para todos los valores de los parámetros.

Demostración: La matriz Jacobiana evaluada en el punto $(0, C)$ es

$$
D Y_{\eta}(0, C)=\left(\begin{array}{cc}
-C(M+Q C) & 0 \\
S C & -S C
\end{array}\right) .
$$

Entonces, $\operatorname{det} D Y_{\eta}(0, C)=S C^{2}(M+Q C)>0 \mathrm{y}$

$\operatorname{tr} D Y_{\eta}(0, C)=-C(M+Q C)-S C<0$.

En consecuencia, el punto de equilibrio $(0, C)$ es atractor. 
3.1.2. Naturaleza de los puntos de equilibrio positivo. Suponiendo que $a_{1}=1+M-Q>0$, entonces las raíces de la ecuación (2.3) dependen del signo de

$$
\Delta=(1+M-Q)^{2}-4(C Q+M)
$$

Caso 1a1. Supongamos $\Delta>0$, etonces existen dos soluciones reales

$$
u_{1}=\frac{1}{2}\left(a_{1}-\sqrt{\Delta}\right) \text { y } u_{2}=\frac{1}{2}\left(a_{1}+\sqrt{\Delta}\right)
$$

con $u_{1}<u_{2}$.

Evaluando la matriz Jacobiana en los puntos de equilibrio positivos, tenemos

$$
D Y_{\eta}(u, u+C)=\left(\begin{array}{cc}
(u+C) u(M-2 u+1) & -Q u(C+u) \\
S(u+C) & -S(u+C)
\end{array}\right),
$$

y $\operatorname{det} D Y_{\eta}(u, u+C)=-S u(C+u)^{2}(M-Q-2 u+1)$, cuyo signo depende del factor

$$
T=M-Q-2 u+1=a_{1}-2 u \text {. }
$$

Además la traza es

$$
\operatorname{tr} D Y_{\eta}(u, u+C)=(u+C)(u(M-2 u+1)-S),
$$

cuyo signo depende del factor

$$
T_{1}=u(M-2 u+1)-S \text {. }
$$

Para determinar si los puntos de equilibrio hiperbólicos son nodos o focos, definimos la cantidad

$$
\begin{aligned}
H & =\left(\operatorname{tr} D Y_{\eta}\left(u_{2}, u_{2}+C\right)\right)^{2}-4 \operatorname{det} D Y_{\eta}\left(u_{2}, u_{2}+C\right) \\
& =((u+C)(u(M-2 u+1)-S))^{2}-4\left(-S u(C+u)^{2}(M-Q-2 u+1)\right) .
\end{aligned}
$$

Teorema 3.1. El equilibrio $P_{1}=\left(u_{1}, u_{1}+C\right)$ es un punto silla [6].

Demostración: Evaluando el factor $T$ evaluando en $u_{1}$ se tiene que

$$
T=a_{1}-2 u_{1}=a_{1}-2\left(\frac{1}{2}\left(a_{1}-\sqrt{\Delta}\right)\right)=\sqrt{\Delta}>0 .
$$

Luego, $\operatorname{det} D Y_{\eta}(u, u+C)<0$.

Por el Teorema de la traza y el determinante $P_{1}=\left(u_{1}, u_{1}+C\right)$ es un punto silla.

Antes de establecer la naturaleza del punto $P_{2}=\left(u_{2}, u_{2}+C\right)$ debemos mostrar algunas propiedades del sistema, relacionadas con las variedades estable e inestable del equilibrio $P_{1}$ y la variedad inestable del punto silla $(1,0)$.

Teorema 3.2. Existencia de una curva homoclínica [13, 18, 38]

Sea $W_{+}^{s}\left(P_{1}\right)$ y $W_{+}^{u}\left(P_{1}\right)$, la variedad estable superior y la variedad inestable derecha del punto silla $P_{1}$, respectivamente. Entonces, existe un subconjunto de valores de parámetros para el que coinciden las dos variedades, formando un lazo homoclínico.

Demostración: Usando el teorema de existencia y unicidad de las soluciones [13] y la geometría de las variedades estable e inestable del punto silla $P_{1}=\left(u_{e 1}, u_{e 1}+C\right)$.

La variedad inestable derecha $W_{+}^{u}\left(P_{1}\right)$ del punto silla $P_{1}$ no puede cruzar la recta hacia la derecha de la recta $u=1$, que es la frontera de la región de invarianza. Por lo tanto, esta solución deben cambiar su dirección y retornar hacia la iquierda.

A su vez, el $\alpha$ - limite de la variedad estable superior $P_{1} W_{+}^{s}\left(P_{1}\right)$ del equilibrio $P_{1}$ puede estar en:

i) el punto $(\infty, 0)$ en el dominio compacto del campo vectorial $Y_{\eta}(u, v)$,

ii) el punto $P_{2}$, cuando este equilibrio es repulsor, o

iii) un ciclo límite inestable rodeando al punto de equilibrio $P_{2}$, cuando este equilibrio es atractor.

Luego, existe condiciones en el espacio de parámetros para los cuales $W_{+}^{s}\left(P_{1}\right) \cap W_{+}^{u}\left(P_{1}\right) \neq \phi$.

Entonces, existe un curva homoclínica, creada por el punto silla $P_{1}$ y rodeando al punto $P_{2}$.

Teorema 3.3. Sean $W_{+}^{u}(1,0)$ la variedad inestable superior del punto silla $(1,0)$ y $W_{+}^{s}\left(u_{e 1}, u_{e 1}+C\right)$ la variedad estable superior del punto $\left(u_{e 1}, u_{e 1}+C\right)$. Consideremos los puntos $\left(u^{*}, v^{u}\right) \in W_{+}^{u}(1,0)$ y $\left(u^{*}, v^{s}\right) \in W_{+}^{u}\left(u_{e 1}, u_{e 1}+C\right)$, con $M<u_{e 1}<u^{*}<1$.

La relación entre $v^{u}$ y $v^{s}$, determina la estabilidad del punto $\left(u_{e 2}, u_{e 2}+C\right)$ de la siguiente manera:

1. Supongamos que $v^{s}>v^{u}$, entonces el punto de equilibrio es:

(a) un atractor hiperbólico, si y solo si, $\left(M-2 u_{e 2}+1\right) u_{e 2}<S$.

(b) un repulsor hiperbólico, si y sólo si, $\left(M-2 u_{e 2}+1\right) u_{e 2}>S$.

(c) un foco débil, si y sólo si, $\left(M-2 u_{e 2}+1\right) u_{e 2}=S$. 
2. Supongamos que $v^{s}<v^{u}$, entonces, entonces el punto de equilibrio $\left(u_{2}, v_{2}\right)$ es

(a) un atractor rodeado por un ciclo límite inestable.

(b) un nodo o foco repulsor hiperbólico y las trayectorias del sistema (2.2) tienen al punto $(0, C)$ como su $\omega$ - limite, el cual es un equilibrio casi globalmente estable [37, 39].

Demostración:

1. Supongamos que $v^{s}>v^{u}$.

Entonces $W_{+}^{s}\left(u_{e 1}, u_{e 1}+C\right)$ la variedad estable superior del punto $\left(u_{e 1}, u_{e 1}+C\right)$, está por encima de $W_{+}^{u}(1,0)$ la variedad estable superior del punto $(1,0)$.

Luego el $\omega$-limite de $W_{+}^{u}(1,0)$ debe ser:

(a) el punto $\left(u_{e 1}, u_{e 1}+C\right)$, originando una curva heteroclínica,

(b) el punto $\left(u_{e 2}, u_{e 2}+C\right)$, cuando es un nodo atractor, o

(c) un ciclo límite atractor.

Evaluando el factor $T$ en el punto $\left(u_{e 2}, u_{e 2}+C\right)$ se tiene que

$$
T=a_{1}-2 u_{2}=a_{1}-2\left(\frac{1}{2}\left(a_{1}+\sqrt{\Delta}\right)\right)=-\sqrt{\Delta}
$$

Luego, $\operatorname{det} D Y_{\eta}(u, u+C)>0$.

Por lo tanto, la naturaleza depende del signo de la traza.

Luego, cuando $T_{1}=u_{e 2}\left(M-2 u_{e 2}+1\right)-S$ es positivo, negativo o cero, se tiene la tesis.

Si la traza es positiva, de acuerdo al Teorema de existencia y unicidad y por el Teorema de PoincaréBendixson, debe existir un ciclo límite rodeando al punto de equilibrio $\left(u_{e 2}, u_{e 2}+C\right)$.

2. Supongamos que $v^{s}<v^{u}$.

Entonces $W_{+}^{s}\left(u_{e 1}, u_{e 1}+C\right)$ la variedad estable superior del punto $\left(u_{e 1}, u_{e 1}+C\right)$, está por debajo de $W_{+}^{u}(1,0)$ la variedad estable superior del punto $(1,0)$.

(a) Si $\left(M-2 u_{e 2}+1\right) u_{e 2}<S$ el punto de equilibrio $\left(u_{e 2}, u_{e 2}+C\right)$ es un atractor hiperbólico. Entonces el $\omega$ - limite de $W_{+}^{s}\left(u_{e 1}, u_{e 1}+C\right)$ es:

i. el punto $\left(u_{e 2}, u_{e 2}+C\right)$, o bien

ii. un ciclo límite atractor rodeando a un ciclo límite inestable y al punto de equilibrio $\left(u_{e 2}, u_{e 2}+C\right)$.

(b) $\mathrm{Si}\left(M-2 u_{e 2}+1\right) u_{e 2}>S$, el punto $\left(u_{e 2}, u_{e 2}+C\right)$ es repulsor (foco o nodo). Entonces, las soluciones con $\alpha$-limite en las vecindades del punto $\left(u_{e 2}, u_{e 2}+C\right)$ tienen como $\omega$-limite:

i. un ciclo límite atractor (o estable), cuando $H<0$ o bien,

ii. el punto $(0, C)$, cuando $H>0$.

Corolario 3.1. Existe una bifurcación de Hopf [18] en el punto de equilibrio $\left(u_{e 2}, u_{e 2}+C\right)$ para el valor de la bifurcación $S=\left(M-2 u_{e 2}+1\right) u_{e 2}$.

Demostración: La demostración sigue del teorema descrito anteriormente, cuando $\operatorname{det} D Y_{\eta}\left(u_{e 2}, u_{e 2}+C\right)=-S u\left(C+u_{e 2}\right)^{2}()$, es positiva y

$\operatorname{tr} D Y_{\eta}\left(u_{e 2}, u_{e 2}+C\right)=\left(u_{e 2}+C\right)\left(u_{e 2}\left(M-2 u_{e 2}+1\right)-S\right)$ cambia de signo..

Además, la condición de transversalidad [13, 20, 38] se verifica ya que

$$
\frac{\partial}{\partial S}\left(\operatorname{tr} D Y_{\eta}\left(u_{e 2}, u_{e 2}+C\right)\right)=-u_{e 2}\left(u_{e 2}+C\right)<0 .
$$

Teorema 3.4. Bifurcación de Bogdanov-Takens [30]

a) El punto $P_{*}=\left(u^{*}, u^{*}+C\right)$, colapso de $P_{1}$ y $P_{2}$ es un silla-nodo atractor.

b) El punto $P_{*}=\left(u^{*}, u^{*}+C\right)$, colapso de $P_{1}$ y $P_{2}$ es un punto cúspide, si y solo si, $\operatorname{tr} D Y_{\eta}\left(u^{*}, u^{*}+C\right)=0 y \operatorname{det} D Y_{\eta}\left(u^{*}, u^{*}+C\right)=0$.

Demostración: a) Como $u^{*}=\frac{1}{2}(1+M-Q)$ y $\operatorname{det} D Y_{\eta}\left(u^{*}, u^{*}+C\right)=-S u^{*}\left(C+u^{*}\right)^{2} T$

con $T=M-Q-2 u^{*}+1$

Es claro que $\operatorname{det} D Y_{\eta}\left(u^{*}, u^{*}+C\right)=0$, cuando colapsan los puntos $P_{1}$ y $P_{2}$, y se tiene que $P_{*}$ es un silla-nodo.

Antes de esta coincidencia, los puntos $P_{1}$ y $P_{2}$ son silla y nodo atractor, respectivamente.

Luego, $P_{*}$ es un silla-nodo atractor [30].

b) Como

$$
\begin{aligned}
T_{1} & =u^{*}\left(M-2 u^{*}+1\right)-S \\
& =u^{*}\left(M-2\left(\frac{1}{2}(1+M-Q)\right)+1\right)-S \\
& =-S+Q u^{*}=-S+Q\left(\frac{1}{2}(1+M-Q)\right) . .
\end{aligned}
$$

Por lo tanto, $P_{*}$ es un punto cúspide. 
4. Efecto Allee débil especial. Cuando el parámetro $M=0$ el sistema queda descrito por

$$
\Gamma_{\eta}(u, v):\left\{\begin{array}{l}
\frac{d u}{d \tau}=((1-u) u-Q v)(u+C) u, \\
\frac{d v}{d \tau}=S(u+C-v) v .
\end{array}\right.
$$

donde $\eta=(Q, S, C) \in \mathbb{R}^{3}$.

Los puntos de equilibrio del sistema (4.1) son: los que estan sobre los ejes $(0,0),(1,0),(0, C)$ y los puntos interiores, obtenidos al intersectar las isoclinas, cuya existencia depende del discriminante de la ecuación de segundo grado

$$
u^{2}-(1-Q)+Q C=0 .
$$

Sea $\triangle_{(2,2)}=(1-Q)^{2}-4 Q C$.

La ecuación (4.2) tiene :

1. Dos raíces reales positivas, si y solo si, $1-Q>0$ y $\triangle_{(2,2)}>0$, obteniendo que $u_{1}=\frac{1-Q-\sqrt{(1-Q)^{2}-4 Q C}}{2}$ y $u_{2}=\frac{1-Q+\sqrt{(1-Q)^{2}-4 Q C}}{2}$.

En este caso obtenemos dos puntos de equilibrios $\left(u_{1}, v_{1}\right)$ y $\left(u_{2}, v_{2}\right)$ donde $v_{i}=u_{i}+C$ con $i=1,2$.

2. Una raíz real positivas, si y solo si, $1-Q>0$ y $\triangle_{(2,2)}=0$,

obteniendose que el punto de equilibrio es $\left(u^{*}, v^{*}\right)=\left(\frac{1-Q}{2}, u^{*}+C\right)$.

3. No tiene raíces reales positivas si $1-Q>0$ y $\triangle_{(2,2)}<0$, o bien $1-Q \leq 0$.

La matriz Jacobiana asociada al sistema (4.1) es:

$$
D \Gamma_{\eta}(u, v)=\left(\begin{array}{cc}
D \Gamma(u, v)_{11} & -Q u(C+u) \\
S v & S(u+C-2 v)
\end{array}\right)
$$

donde

$$
D \Gamma(u, v)_{11}=-4 u^{3}+3(1-C) u^{2}+2(C-Q v) u-C Q v .
$$

El sistema (4.1) tiene las siguientes propiedades:

Lema 4.1. El punto de equilibrio $(1,0)$ es silla hiperbólica para todos los valores de los parámetros.

Demostración: Como $D \Gamma_{\eta}(1,0)=\left(\begin{array}{cc}-(C+1) & -Q(C+1) \\ 0 & S(C+1)\end{array}\right)$, tenemos que

$\operatorname{det} D \Gamma_{\eta}(1,0)=-S(C+1)^{2}<0$.

Luego $(1,0)$ es un punto silla.

Lema 4.2. El punto de equilibrio $(0, C)$ es nodo atractor hiperbólico para todos los valores de los parámetros.

$$
\begin{aligned}
& \text { Demostración: Como } D \Gamma_{\eta}(0, C)=\left(\begin{array}{cc}
-C^{2} Q & 0 \\
S C & -S C
\end{array}\right) \text {, entonces } \\
& \quad \operatorname{det} D \Gamma_{\eta}(0, C)=C^{3} Q S>0, \operatorname{tr} D \Gamma_{\eta}(0, C)=-\left(C^{2} Q+C S\right)<0, \mathrm{y} \\
& \triangle=\left(-C^{2} Q-C S\right)^{2}-4 C^{3} Q S \triangle=C^{2}(S-C Q)^{2}>0 .
\end{aligned}
$$

Luego $(0, C)$ es nodo atractor.

Lema 4.3. El punto de equilibrio $(0,0)$ es un silla-nodo (no- hiperbólico) para todos los valores de los parámetros.

Demostración: En este caso $M=0$, el punto $(0,0)$ es un colapso del punto $(0,0)$ y el punto $(M, 0)$ cuya naturaleza en el caso $M>0$ es silla y nodo repulsor respectivamente, por lo tanto en este caso es un silla-nodo.

Lema 4.4. El punto de equilibrio $\left(u_{1}, v_{1}\right)$ es un punto silla para todos los valores de los parámetros.

Demostración: Análoga a la demostración de Teorema 3.1 
Teorema 4.1. Sean $W_{+}^{u}(1,0)$ la variedad inestable superior del punto silla $(1,0)$ y $W_{+}^{s}\left(u_{e 1}, u_{e 1}+C\right)$ la variedad estable superior del punto $\left(u_{e 1}, u_{e 1}+C\right)$. Consideremos los puntos $\left(u^{*}, v^{u}\right) \in W_{+}^{s}(1,0)$ y $\left(u^{*}, v^{s}\right) \in W_{+}^{u}\left(u_{e 1}, u_{e 1}+C\right)$, con $0<u_{e 1}<u^{*}<1$.

De acuerdo a la relación entre $v^{u} y v^{s}$, tenemos la naturaleza del punto $\left(u_{e 2}, u_{e 2}+C\right)$ de la siguiente manera:

1. Supongamos que $v^{s}>v^{u}$, entonces el punto de equilibrio es:

1.1 un atractor hiperbólico, si y solo si, $S>\left(-2 u_{e 2}+1\right) u_{e 2}$.

1.2 un repulsor hiperbólico, si y sólo si, $S<\left(-2 u_{e 2}+1\right) u_{e 2}$.

1.3 un foco débil, si y sólo si, $S=\left(-2 u_{e 2}+1\right) u_{e 2}$.

2. Supongamos que $v^{s}<v^{u}$, entonces, entonces el punto de equilibrio $\left(u_{2}, v_{2}\right)$ es

2.1 un atractor rodeado por un ciclo límite inestable.

2.2 un nodo o foco repulsor hiperbólico y las trayectorias del sistema (4.1) tienen al punto $(0, C)$, como su $\omega$ - limite, el cual es un equilibrio casi globalmente estable [37, 39].

Demostración: Similar a la demostración del Teorem 3.3 $\left(u_{2}, v_{2}\right)$.

En el caso $1-Q>0$ y $\triangle_{(2,2)}=0$ tenemos el punto $\left(u^{*}, v^{*}\right)$, que es el colapso de los puntos $\left(u_{1}, v_{1}\right)$ y

Teorema 4.2. Bifurcación de Bogdanov-Takens [30]

El punto $\left(u^{*}, v^{*}\right)$ es

1. silla-nodo repulsor, si y solo si, $(C Q(Q+1)-S(2 C-Q+1))>0$,

2. silla-nodo atractor, si y solo si, $(C Q(Q+1)-S(2 C-Q+1))<0$,

3. punto cúspide. si y solo si, $(C Q(Q+1)-S(2 C-Q+1))=0$.

Demostración:

La matriz jacobiana evaluada en el punto es

$$
u^{*}=\frac{1-Q}{2} \quad \text { y } \quad v^{*}=u^{*}+C .
$$

$$
D \Gamma_{\eta}\left(u^{*}, v^{*}\right)=\left(\begin{array}{cc}
\frac{1}{2} C Q(Q+1) & -\frac{1}{2} C Q(Q+1) \\
\frac{1}{2} S(1-Q+2 C) & -\frac{1}{2} S(1-Q+2 C)
\end{array}\right) .
$$

Luego

$\operatorname{det} D \Gamma_{\eta}\left(u^{*}, v^{*}\right)=0$ y $\operatorname{tr} D \Gamma_{\eta}\left(u^{*}, v^{*}\right)=\frac{1}{2}(C Q(Q+1)-S(2 C-Q+1))$. de la traza.

Este punto es colapso de los puntos $\left(u_{1}, v_{1}\right)$ y $\left(u_{2}, v_{2}\right)$. El primero es siempre silla y el segundo depende

Por lo tanto $\left(u^{*}, v^{*}\right)$ es un silla-nodo [30].

Analizando el signo de la traza y despejando $S$, tenemos los resultados enunciados.

5. Efecto Allee débil. Ahora, veremos el caso en que consideramos $M<0$ en el sistema (2.2).

Teorema 5.1. Para este caso tenemos los siguientes resultados

1. El punto de equilibrio $(0,0)$ es un nodo repulsor hiperbólico,

2. El punto $(1,0)$ es punto silla hiperbólica,

3. El punto $(0, C)$ es:

- silla hiperbólica, si y solo si, $(M+C Q)<0$,

- nodo atractor hiperbólico, si y solo si, $(M+C Q)>0$.

Demostración:

1. La matriz jacobiana evaluada en el punto $(0,0)$ es:

entonces

$$
D \Gamma_{\eta}(0,0)=\left(\begin{array}{cc}
-M C & 0 \\
0 & S C
\end{array}\right),
$$

$\operatorname{det} D \Gamma_{\eta}(0,0)=-M C^{2} S>0 \mathrm{y} \operatorname{tr} D \Gamma_{\eta}(0,0)=(-M+S) C>0$.

Luego, el discriminante de la ecuación característica

$\triangle=(-C M+C S)^{2}+4 C M S=C^{2}(M+S)^{2}>0$,

Por tanto, obtenemos que $(0,0)$ es un nodo repulsor.

2. La matriz Jacobiana evaluada en el punto $(1,0)$ es

$$
D \Gamma_{\eta}(1,0)=\left(\begin{array}{cc}
-(C+1)(1-M) & -Q(C+1) \\
0 & S(C+1)
\end{array}\right) .
$$


y dado que $(1-M)>0$, tenemos que

$\operatorname{det} D \Gamma_{\eta}(1,0)=-S(C+1)^{2}(1-M)<0$.

Por lo tanto el punto de equilibro $(1,0)$ es un punto silla hiperbólica.

3. La matriz Jacobiana evaluada en el punto $(0, C)$ es

$$
D \Gamma_{\eta}(0, C)=\left(\begin{array}{cc}
-\left(M C+C^{2} Q\right) & 0 \\
S C & -S C
\end{array}\right) .
$$

Si $(M+C Q)<0$ tenemos que

$\operatorname{det} D \Gamma_{\eta}(0, C)=(M+C Q) S C^{2}<0$,

luego el punto de equilibrio $(0, C)$ es silla hiperbólica.

$\mathrm{Si}(M+C Q)>0$ entonces

$\operatorname{det} D \Gamma_{\eta}(0, C)=(M+C Q) S C^{2}>0$,

$\operatorname{Tr} D \Gamma_{\eta}(0, C)=\left(-\left(M C+C^{2} Q\right)-S C\right)=-C(M+C Q+S)<0$ y

$\triangle=(-C(M+C Q+S))^{2}+4\left((M+C Q) S C^{2}\right)=C^{2}(M-S+C Q)^{2}>0$.

Por lo tanto tenemos que el punto de equilibrio $(0, C)$ es nodo atractor.

Observación 5.1. Para tener la existencia de los puntos de equilibrio positivos, en el caso $M<0$ debemos analizar el signo de $M+C Q$ en la ecuación (2.3).

a) Si $M+C Q>0$ entonces tenemos los mismos casos que cuando $M>0$ y la naturaleza de los puntos de equilibrio es similar al caso en que $M>0$.

b) Si $M+C Q<0$ entonces por el apartado 3.3 el sistema (2.2) tiene un único punto de equilibrio positivo $\left(u_{6}, u_{6}+C\right) o\left(u_{7}, u_{7}+C\right)$.

Como en este caso el punto de equilibrio $(0, C)$ es silla hiperbólica, entonces el único punto de equilibrio positivo en la región de invarianza $\tilde{\Gamma}$ puede ser:

b1) un atractor global asintóticamente etable (gas) [13, 20, 30, 38].

b2) un punto repulsor rodeado de un ciclo límite estable, por Teorema de Poicaré-Bendixson, o

b3) un atractor local, rodeado de dos ciclos límtes concéntricos, el interior inestable y el exterior estable, es decir, exixte una Bifurcación de Hopf múltiple y el punto de equilibrio positivo es un foco débil de orden 2 [13, 20,30,38].

Además el punto $(M, 0)$ está en el segundo cuadrante y no tiene interés ecológico.

6. Conclusiones. Los modelos matemáticos son una herramienta fundamental para comprender y analizar la dinámica de los cadenas alimenticias. En ecología de poblaciones, un tema clásico y significativo es la interacción entre depredadores y sus presas. que son una parte importante de esas cadenas. Por ese motivo, en los últimos años, los modelos de depredación del tipo Leslie han recibido mayor atención para investigar los comportamientos dinámicos entre las especies.

En este trabajo hemos mostrado las principales propiedades de un modelo de depredación tipo LeslieGower, asumiendo que los depredadores tienen un alimento alternativo, en el caso de que su presa favorita sea escasa; además se ha asumido que la población de presas es afectada por el fenómeno denominado efecto Allee [8, 10, 11, 33] .

Mediante un difeomorfismo obtuvimos un sistema topológicamente equivalente al original, dependiente sólo de cuatro parámetros. Demostramos que en este modelo (y por tanto en el original) existen diferentes situaciones dinámicas según las relaciones obtenidas entre los parámetros.

Entre los resultados más importantes, se ha demostrado la existencia de una curva separatriz $\bar{\Sigma}$, dividiendo el comportamiento de las soluciones o trayectorias del sistema en el plano de fase. Esta curva separatriz existe cuando hay dos puntos de equilibrio positivos al interior del primer cuadrante. Es generada por la variedad estable superior $W_{+}^{s}\left(u_{e 1}, u_{e 1}+C\right)$ del punto silla $\left(u_{e 1}, u_{e 1}+C\right)$. Esto implica que para el mismo conjunto de parámetros, dos soluciones muy cercanas pero en un lado diferente de esa separatriz $\bar{\Sigma}$, tendrían $\omega$-limites diferentes y lejanos, lo cual significa que las soluciones son altamente sensibles a las condiciones iniciales.

Nuestros resultados también confirman el hecho de que si la población de depredadores es generalista, la extinción de cada especie puede evitarse, para ciertas condiciones iniciales [24, 25, 28, 36].

El modelo estudiado tiene una rica e interesante dinámica. como otros modelos del tipo Leslie-Gower modificado la cual se destaca cuando se compara con el modelo del tipo Leslie-Gower donde la tasa de crecimiento de las presas es del tipo logístico y los depredadores tienen también un alimento alternativo, pero en el que el efecto Allee está ausente. En este simple modelo exite un único punto de equilibrio positivo que es globalmente asintótico estable (gas) lo cual se prueba construyendo funciones de Lyapunov adecuadas [27, 29].

Una extención de este trabajo debería enfocarse en establecer el número de ciclos límites, que rodean un punto de equilibrio positivo del tipo de centro.foco, originados por bifurcaciones Hopf múltiples [13, 20, 21, 26]. 
Esta no es una tarea fácil y está relacionada con la resolución del conocido problema 16 de Hilbert (propuesto por el matemático alemán David Hilbert en 1900), el cual se refiere al número máximo y la posición relativa de los ciclos límites en los sistemas de ecuaciones diferenciales bidimensionales [15, 20].

Sin embargo, esta pregunta sigue sin resolverse en el caso del sistema de ecuación diferencial polinómica, cuyo grado debe ser menor o igual que $p \in \mathbb{N}$ con $p \geq 2$ [20].

Particularmente, para los modelos depredador-presa, este problema también permanece sin respuesta. El estudio de este problema comienza con el trabajo de Kuo-Shung Cheng en 1981 [12], quien fue el primero en demostrar la unicidad de un ciclo límite para un modelo específico depredador-presa del tipo Gause [19] considerando una respuesta funcional de Holling tipo II [15].

Consideramos que la determinación del número de ciclos límite que se bifurcan desde un foco débil $[13,30,38]$, es un buen criterio para la clasificación de los modelos depredador-presa.

Además el modelo analizado, satisface las recomendaciones sugeridas en [9] por ser creible, simple y útil.

ORCID and License

Nicole Martínez-Jeraldo https://orcid.org/0000-0002-2267-2057

Elizabeth Rozas-Torres https://orcid.org/0000-0002-8204-2938

Eduardo González-Olivares https://orcid.org/0000-0003-3907-0076

This work is licensed under the Creative Commons - Attribution 4.0 International (CC BY 4.0)

\section{Referencias}

[1] Aguirre P, González-Olivares E, Sáez E. Two limit cycles in a Leslie-Gower predator-prey model with additive Allee effect, Nonlinear Analysis: Real World Applications. 2009; 10:1401-1416.

[2] Aguirre P, González-Olivares E, Sáez E. Three limit cycles in a Leslie-Gower predator-prey model with additive Allee effect, SIAM Journal on Applied Mathematics. 209; 69(5):1244-1269.

[3] Andronov AA, Leontovich EA, Gordon I, Maier AG. Qualitative theory of second-order dynamics systems, A Halsted Press Book, John Wiley \& Sons; 1973.

[4] Arancibia-Ibarra C, González-Olivares E. A modified Leslie-Gower predator-prey model with hyperbolic functional response and Allee effect on prey, In Mondaini R (Ed.), BIOMAT 2010 International Symposium on Mathematical and Computational Biology, Singapore: World Scientific Co. Pte. Ltd., 2011. pp 146-162.

[5] Arancibia-Ibarra C. The basins of attraction in a modified May-Holling-Tanner predator-prey model with Allee effect, Nonlinear Analysis. 2019; 185:15-28.

[6] Arrowsmith DK, Place CM. Dynamical System. Differential equations, maps and chaotic behaviour. Chapman and Hall; 1992.

[7] Bazykin AD. Nonlinear Dynamics of interacting populations, World Scientific Publishing Co. Pte. Ltd., 1998.

[8] Berec L, Angulo E, Courchamp F. Multiple Allee effects and population management. Trends in Ecology and Evolution. 2007; 22:185-191.

[9] Berryman AA, Gutierrez AP, Arditi R. Credible, parsimonious and useful predator-prey models - A reply to Abrams, Gleeson and Sarnelle. Ecology. 1995; 76:1980-1985.

[10] Boukal DS, Berec L. Single-species models and the Allee effect: Extinction boundaries, sex ratios and mate encounters. J. of Theoret. Biology. 2002; 218:375-394.

[11] Boukal DS, Sabelis MW, Berec L. How predator functional responses and Allee effects in prey affect the paradox of enrichment and population collapses. Theoret. Population Biology; 2007; 72:136-147.

[12] Cheng K-S. Uniqueness of a limit cycle for a predator-prey system. SIAM J. on Math. Anal. 1981; 12:541-548.

[13] Chicone C. Ordinary differential equations with applications. (2nd edition), Texts in Applied Mathematics 34. New York: Springer; 2006.

[14] Clark CW. Mathematical Bioeconomic: The optimal management of renewable resources, (second edition). New York: John Wiley and Sons; 1990.

[15] Coleman CS. Hilbert's 16th. Problem: How Many Cycles? In: M. Braun, CS. Coleman and D. Drew (Ed). Differential Equations Model, Springer Verlag; 1983; pp 279-297.

[16] Courchamp F, Clutton-Brock T, Grenfell B. Inverse density dependence and the Allee effect, Trends in Ecology and Evolution. 1999; 14(10):405-410.

[17] Courchamp F, Berec L, Gascoigne J. Allee effects in Ecology and Conservation, Oxford University Press 2007.

[18] Dumortier F, Llibre J, Artés JC. Qualitative theory of planar differential systems, Springer, 2006.

[19] Freedman HI. Deterministic Mathematical Model in Population Ecology, Marcel Dekker New York 1980.

[20] Gaiko VA. Global Bifurcation theory and Hilbert's sixteenth problem, Mathematics and its Applications 559, Kluwer Academic Publishers New York 2003.

[21] Gaiko VA, Vuik V. Global dynamics in the Leslie-Gower model with the Allee effect, Int. J. of Bifurcation and Chaos. 2018 , Vol. 28(12): 1850151 (10 pages).

[22] Goh B-S. Management and Analysis of Biological Populations. Elsevier Scientific Publishing Company; 1980.

[23] González-Olivares E, González-Yañez B, Mena-Lorca J, Ramos-Jiliberto R. Modelling the Allee effect: are the different mathematical forms proposed equivalents?, In Mondaini R (Ed.), Proceedings of the 2006 International Symposium on Mathematical and Computational Biology, Rio de Janeiro; E-papers Serviços Editoriais Ltda. 2007; pp 53-71.

[24] González-Olivares E, Mena-Lorca J, Rojas-Palma A, Flores JD. Dynamical complexities in the Leslie-Gower predator-prey model as consequences of the Allee effect on prey. Applied Mathematical Modelling. 2011; 35:366-381.

[25] González-Olivares E, Mena-Lorca J, Rojas-Palma A, Flores JD. Erratum to Dynamical complexities in the Leslie-Gower predator- 
prey model as consequences of the Allee effect on prey. [Appl. Math. Modell. (2011) 366-381], Applied Mathematical Modelling, 2012; 36:860-862.

[26] González-Olivares E, Rojas-Palma A, González-Yañez B. Multiple limit cycles in a Leslie-Gower type predator-prey model considering weak Allee effect on prey, Nonlinear Analysis: Modelling and Control. 2017; 22(3):347-365.

[27] González-Olivares E, Rojas-Palma A. Global stability in a modified Leslie-Gower type predation model assuming mutual interference among generalist predators. Math. Biosciences and Eng. 2020; 17(6):7708-7731.

[28] González-Yañez B, González-Olivares E, Mena-Lorca J. Multistability on a Leslie-Gower type predator-prey model with nonmonotonic functional response, In Mondaini R, Dilao R (Eds.), BIOMAT 2006 - International Symposium on Mathematical and Computational Biology, World Scientific Co. Pte. Ltd. 2007. pp 359-384.

[29] Korobeinikov A. A Lyapunov function for Leslie-Gower predator-prey models. Applied Mathematical Letters. 2001; 14:697-699.

[30] Kuznetsov YA. Elements of applied bifurcation theory. (3rd edition), Springer; 2004.

[31] Leslie PH. Some further notes on the use of matrices in population mathematics, Biometrica. 1948; 35:213-245.

[32] Leslie PH, Gower JC. The properties of a stochastic model for the predator-prey type of interaction between two species. Biometrica; 1960; 47:219-234.

[33] Liermann M, Hilborn R. Depensation: evidence, models and implications. Fish and Fisheries; 2001; 2:33-58.

[34] Martínez-Jeraldo N, Aguirre P. Allee effect acting on the prey species in a Leslie-Gower predation model. Nonlinear Analysis: Real World Applications; 2019; 45:895-917.

[35] May RM. Stability and complexity in model ecosystems (2nd edition), Princeton: Princeton University Press; 2001.

[36] Mena-Lorca J, González-Olivares E, González-Yañez B. The Leslie-Gower predator-prey model with Allee effect on prey: A simple model with a rich and interesting dynamics, in: Mondaini R. (Ed.), Proceedings of the 2006 International Symposium on Mathematical and Computational Biology BIOMAT 2006, Rio de Janeiro: E-papers Serviços Editoriais Ltda. 2007. pp $105-132$.

[37] Monzón P. Almost global attraction in planar systems, Systems \& Control Letters, 2005; 54:753-758.

[38] Perko L. Differential equations and dynamical systems (3rd edition), Springer-Verlag, 2001.

[39] Rantzer A. A dual to Lyapunov's stability theorem, Systems and Control Letters; 2001; 42(3):161-168.

[40] Sáez E, González-Olivares E. Dynamics on a Predator-prey Model, SIAM J. of Applied Math. 1999; 59(5):1867-1878.

[41] Stephens PA, Sutherland WJ. Consequences of the Allee effect for behaviour, ecology and conservation, Trends in Ecology and Evolution, 1999; 14:401-405.

[42] Stephens PA, Sutherland WJ, Freckleton RP. What is the Allee effect?, Oikos. 1999; 87:185-190.

[43] Turchin P. Complex population dynamics. A theoretical/empirical synthesis, Monographs in Population Biology 35. Princeton: Princeton University Press, 2003.

[44] van Voorn GAK, Hemerik L, Boer MP, Kooi BW. Heteroclinic orbits indicate overexploitation in predator-prey systems with a strong Allee. Math. Biosciences. 2007; 209:451-469.

[45] Volterra V. Variazioni e fluttuazioni del numero d'individui in specie animali conviventi. Memorie della R. Accademia dei Lincei, S.VI, IT. 1926; II; 31-113.

[46] Wang J, Shi J, Wei J, Predator-prey system with strong Allee effect in prey. J. of Mathematical Biology; 2011; 62:291-331. 